\title{
Simultaneous optical and infrared polarimetry of blazars with TRISPEC/KANATA
}

\author{
M. Uemura*, M. Sasada, A. Arai, Y. Fukazawa, K. S. Kawabata, T. Ohsugi, \\ T. Yamashita, M. Isogai, T. Mizuno, H. Katagiri ${ }^{\dagger}$ \\ Hiroshima University, Kagamiyama 1-3-1, Higashi-Hiroshima, 739-8526, Japan \\ E-mail: uemuramehiroshima-u.ac.jp
}

\section{S. Sato, M. Kino}

Nagoya University, Furo-cho, Chikusa-ku, Nagoya, Japan

\begin{abstract}
Owing to the development of the Internet service, studies of astronomical transient phenomena have entered a new era, in which we can promptly receive and send the information about transients and variables. On the other hand, a sudden requirement for dense and long monitorings for them is still difficult for large telescopes. Here, we introduce our new observatory and telescope, which are dedicated for studies of various kinds of transients, including blazars. Our 1.5-m telescope "KANATA" was installed at Higashi-Hiroshima Observatory in May 2006. Using unique instruments attached to KANATA, we can study transients in near-infrared wavelengths, with polarimetry, and with high-speed photometry. In this article, we show a part of our results about 3C 454.3 .
\end{abstract}

Workshop on Blazar Variability across the Electromagnetic Spectrum

April 22-25 2008

Palaiseau, France

\footnotetext{
* Speaker.

$\dagger$ This work was partly supported by a Grand-in-Aid from the Ministry of Education, Culture, Sports, Science, and Technology of Japan (19740104).
} 


\section{The KANATA telescope}

Astronomical transient phenomena have received much attention since ancient times. During the last decade, observations of transients have experienced a revolution led by the development of the Internet. We can now send the information of variables to general public, immediately receive them, and quickly start observations. On the other hand, the revolution was, in general, only for small, 30-cm class telescopes, since only few observation times were allowed for transients in large telescopes. Most recently, however, the situation has been changing. Due to the development of 8-10-m class telescopes, 1-m class telescopes are now regarded as "small" ones, having a plenty of flexible observation times. They have a great potential for observations of transients.

Here, we introduce our project, "Higashi-Hiroshima Observatory". We moved a 1.5-m telescope installed in Tokyo in 1995 by National Astronomical Observatory of Japan to our new observatory in Hiroshima. In 2006 May, our new observatory opened, and the telescope was given a new name, "KANATA" (having a meaning of "far away" in Japanese). This is the telescope dedicated for astronomical transients. Owing to a plenty of observation times and an easy access to the observatory (20-min by car from our office), we have obtained several results for $\gamma$-ray bursts (GRBs), blazars, supernovae, X-ray binaries, cataclysmic variables, and others [1][2][3][4][5].

As a 1-m class telescope, KANATA is one of ordinary telescopes, having Ritchey-Chretien optics and three available focuses (one Cassegrain and two Nasmith), except for its high driving speed. The azimuth and altitude driving speeds are $5^{\circ}$ and $2^{\circ} \mathrm{s}^{-1}$, respectively. This high motion is required to obtain very early light curves and polarizations of GRBs and their afterglows.

At the Cassegrain focus, TRISPEC (Triple Range Imager and SPECtrograph), developed by Nagoya University, is currently attached [6]. In TRISPEC, the incident light is divided into optical $(0.45-0.90 \mu \mathrm{m})$ and two infrared lights $(0.90-1.85$ and $1.85-2.5 \mu \mathrm{m})$ by two dichroic mirrors. Each path has wheels including grisms and Wollaston prisms. TRISPEC, thus, works as a simultaneous optical and near-infrared imager, spectrograph, and polarimeter.

At the 1st Nasmith focus, High Speed Camera is attached, as a collaboration with the Kyoto University team. It has a frame transfer CCD chip, enabling the fastest time-resolution of $0.03 \mathrm{~s}$. We use this camera for X-ray binaries and cataclysmic variables, in which the gas rapidly rotates around compact objects. Its spectroscopic mode is now under development.

For the 2nd Nasmith focus, we are now developing a new instrument, "HOWPol" (Hiroshima One-shot Wide-field Polarimeter), which focuses on rapid variations of polarizations. The operation of HOWPol will start in 2008. A new infrared—optical camera is also under development. It will be the next instrument to replace TRISPEC.

\section{Optical and near infrared photo-polarimetric observation of $3 \mathrm{C} \mathbf{4 5 4 . 3}$}

In this section we show an example of our photo-polarimetric monitoring of blazars. 3C 454.3 is a famous blazar, which had exhibited no major optical flares before 2001, and then entered an active phase [7]. We performed monitoring of 3C 454.3 from Jul 2007 to Jan 2008. Figure 1 shows the light curve and the temporal evolution of polarization parameters on the Stokes $Q-U$ plane obtained with TRISPEC/KANATA. The object was in an outburst in Jul-Aug 2007, and then, faded to its quiescent level. After that, it showed a renewed activity, which can be described with 
repeating short flares having a time-scale of a few days, superimposed on a gradually brightening trend.

The polarization vector experienced clockwise rotation around the maximum of the outburst in JD 2454300-2454350, as can be seen in figure 1. Between JD 2454370 and 2454500, on the $Q-U$ plane, we can see that the short flares have their specific polarization vectors, and furthermore, their vector was apparently rotating anticlockwise with time. These episodes of the rotation of the polarization vector may indicate a moving of the emitting region through a helical magnetic field in a jet.

\section{Summary and Future Plan}

KANATA can provide unique data of transients by infrared, polarimetric, and high speed observations. The long monitoring with the photo-polarimetric mode, in particular, can be a great tool for studies of blazars, as shown in figure 1. From August 2008, we are planning to perform observational campaign for blazars, collaborating with the GLAST satellite. Preliminary results of blazars and other transients will be reported in our web site ${ }^{1}$

\section{References}

[1] Uemura, M., Arai, A., Krajci, T., Pavlenko, E., Shugarov, S. Y., Katysheva, N. A., Goranskij, V. P., Maehara, H., et al. 2008, PASJ, 60, 227

[2] Uemura, M. and Arai, A. and Sasada, M. and Schmeer, P. and Miller, I. and Ohsugi, T. and Yamashita, T. and Kawabata, et al. 2008, IBVS, 5815

[3] Kawabata, K. S. and Sasada, M. and Uemura, M. and Yamashita, T. and Ohsugi, T. and Lundock, R. G. 2008, GCN Circ. 7686

[4] Arai, A. and Uemura, M. and Sasada, M. and Kawabata, K. S. and Yamashita, T. and Yasuda, T. and Matsui, R. and Tanaka, H. et al. 2007, Central Bureau Electronic Telegrams, 1118, 2

[5] Uemura, M. and Sasada, M. and Arai, A. and Uehara, T. 2007, GCN Circ. 7094

[6] Watanabe, M., Nakaya, H., Yamamuro, T., Zenno, T., Ishii, M., Okada, M., Yamazaki, A., Yamanaka, Y., et al. 2005, PASP, 117, 870

[7] Villata, M. and Raiteri, C. M. and Balonek, T. J. and Aller, M. F. and Jorstad, S. G. and Kurtanidze, O. M. and Nicastro, F. and Nilsson, K. 2006, A\&A, 453, 817

\footnotetext{
${ }^{1}$ http://kanatatmp.blogspot.com/
} 

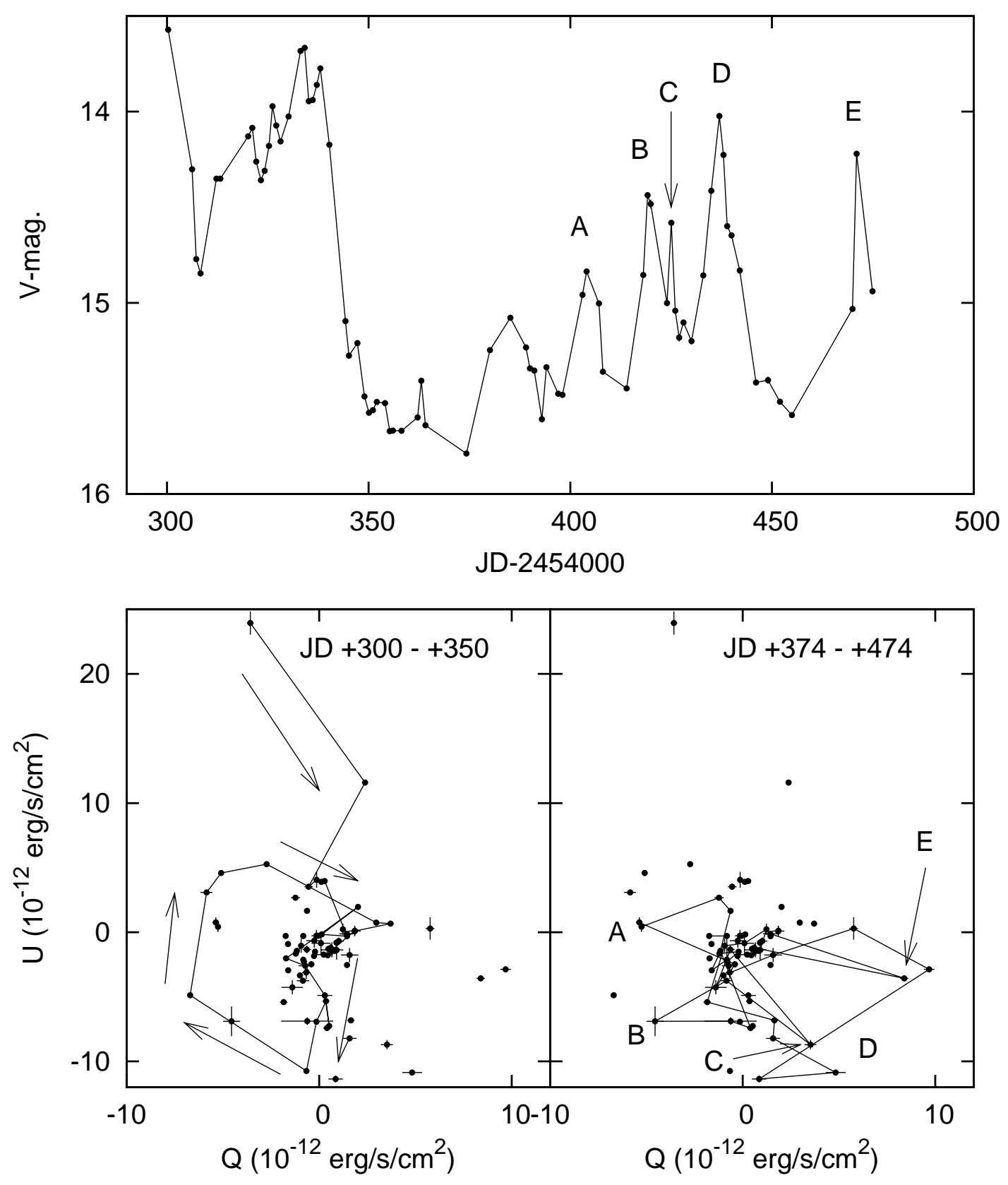

Figure 1: (Upper panel) Light curve of 3C 454.3 in 2007. (Lower panel) Temporal variations of the polarization parameters on $Q-U$ plane. 